\title{
Co-Designed Exposure Protocol in the Study of Idiopathic Environmental Intolerance Attributed to Electromagnetic Fields
}

\author{
Maryse Ledent $\mathbb{D},{ }^{1 *}$ Benjamin Vatovez, ${ }^{2}$ Willy Pirard, ${ }^{2}$ Jimmy Bordarie, ${ }^{3}$ \\ Nicolas Prignot, ${ }^{4}$ Gunnhild Oftedal, ${ }^{5}$ Christophe Geuzaine, ${ }^{6}$ Véronique \\ Beauvois, ${ }^{6}$ Catherine Bouland, ${ }^{7}$ Luc Verschaeve, ${ }^{1}$ and Maël Dieudonné $\mathbb{D}^{8}$ \\ ${ }^{1}$ Sciensano, Brussels, Belgium \\ ${ }^{2}$ Institut Scientifique de Service Public, Liège, Belgium \\ ${ }^{3}$ Quality of Life and Psychological Health, University Institute of Technology of \\ Tours (IUT Tours), Tours, France \\ ${ }^{4}$ Université Libre de Bruxelles, Brussels, Belgium \\ ${ }^{5}$ Department of Electronic Systems, Faculty of Information Technology and \\ Electrical Engineering, NTNU Norwegian University of Science and Technology, \\ Trondheim, Norway \\ ${ }^{6}$ Applied and Computational Electromagnetics Unit, School of Engineering, \\ University of Liège, Liège, Belgium \\ ${ }^{7}$ Ecole de Santé publique, Université Libre de Bruxelles, Brussels, Belgium \\ ${ }^{8}$ Max Weber Center, Institut des Sciences de l'Homme, Lyon, France
}

\begin{abstract}
The hypothesis of an electromagnetic origin of idiopathic environmental intolerance (IEI) attributed to electromagnetic fields (EMF) has been widely investigated by provocation studies, which consist of deliberately exposing people with IEI-EMF in laboratory settings to particular EMF to observe volunteers' reactions. In the majority of these studies, reactions have been found to be independent of exposure. However, most of these studies suffer from design and methodological limitations that might bias their findings or reduce their precision. As provocation studies are best suited for isolating the effects of EMF, innovative protocols should be applied. In the ExpoComm project (PNREST Anses, EST/2017/2 RF/19), several innovations have been introduced: the involvement of people with IEI-EMF in the development of the protocol, the attenuation of the anxiogenic nature of the tests, the individualization of the protocol, the validation of the neutral or normal reactivity state before the test, and the use of a cocktail of real, rather than artificially generated, sources. The objective of involving people with IEI-EMF was to increase the relevance and acceptability of the protocol, while respecting technical constraints and scientific quality requirements. This paper describes the protocol resulting from the collaborative process. Bioelectromagnetics. (C) 2020 Bioelectromagnetics Society
\end{abstract}

Keywords: idiopathic environmental intolerance attributed to electromagnetic fields; exposure protocol; symptoms; field perception; ECG

\section{INTRODUCTION}

Idiopathic environmental intolerance attributed to electromagnetic fields (IEI-EMF) [World Health Organization, 2007], also known as electrohypersensitivity (EHS), is defined by three criteria (i) the perception of various symptoms (fatigue, headaches, concentration difficulties, dermatological, digestive problems, etc.); (ii) the absence of clinical and biological evidence to explain these symptoms; and (iii) their attribution to EMF by the persons concerned [World Health Organization, 2007; Anses, 2018]. The hypothesis of an electromagnetic origin of such symptoms has been widely investigated by provocation
Grant sponsor: PNREST Anses; grant number: EST/2017/2 RF/19 (ExpoComm project).

Conflicts of interest: None.

*Correspondence to: Maryse Ledent, Sciensano, Rue Juliette Wytsmanstraat 14, 1050 Brussels, Belgium.

E-mail: maryse.ledent@ sciensano.be

Received for review 4 March 2020; Accepted 14 June 2020

DOI:10.1002/bem.22281

Published online in Wiley Online Library (wileyonlinelibrary.com). 
studies, which consist of deliberately exposing people with IEI-EMF to particular EMF sources in laboratory settings to observe the volunteers' reactions. In the majority of studies, these reactions have been found to be independent of exposure: people with IEI-EMF did not perceive EMF beyond chance, did not get more symptoms during real rather than sham exposures [Rubin et al., 2010; Schmiedchen et al., 2019], and did not react physiologically (heart rate variability, skin conductivity, cognitive functioning, etc.) differently to real and sham exposure [Rubin et al., 2011; Andrianome et al., 2017]. However, the symptoms of people with IEI-EMF were frequently correlated with perceived exposures in experimental settings [Huss et al., 2016; Andrianome et al., 2017; Verrender et al., 2018] as well as observational studies [Baliatsas et al., 2015; Bolte et al., 2015]. It has thus been suggested that their expectations (conscious or not) of the adverse consequences of EMF exposures may give rise to symptoms [Regel et al., 2006; Oftedal et al., 2007; Rubin et al., 2010]. Nonetheless, such nocebo effects should not be regarded as the only explanation for IEI-EMF symptoms. They are not exclusive of other causes, as established by qualitative studies of the trajectories of people with IEI-EMF, consistently demonstrating that they start feeling sick before becoming suspicious of EMF [de Graaff and Bröer, 2012; Dieudonné, 2016, 2019]. The nocebo hypothesis, as such, does not disprove the existence of a physical effect of EMF exposure.

On the basis of a critical review of provocation studies, Schmiedchen et al. [2019] found that there are just a few high-quality studies and that most studies suffer from design and methodological limitations that might bias their findings or reduce their accuracy. The most common issues relate to the selection of study volunteers in relation to the symptoms being observed and exposure parameters, e.g. some studies did not ensure that the exposure duration and/or observation period was sufficiently long for symptoms to occur, according to the volunteers' descriptions of their usual responses to EMF exposure. An improper control of blinding and a lack of counterbalancing in cross-over studies, along with small numbers of volunteers and lack of statistical power estimates, were other shortcomings in some studies. Some of these criticisms are shared by people with IEI-EMF, as observed in a qualitative survey in Belgium [Prignot, 2020]. Indeed, the results of provocation studies appear incomprehensible to them as they carried out seemingly objective experiments and measurements before concluding that they are hypersensitive [Dieudonné, 2016, 2019]. The assertion that the electromagnetic etiology is not established is perceived by people with
IEI-EMF as a challenge to what they see as the very reality of their disorders, i.e. their somatic nature [Prignot, 2020]. Genuis and Lipp [2012] provide several additional explanations for the negative results of provocation studies, such as the diversity of individual responses to exposures or their short-term variability (based on previous exposure, emotional state, therapies followed, etc.). Similarly, McCarty et al. [2011] suggested that individuals with IEI-EMF respond more to changes in state, i.e., transitions between real and simulated exposure, than to the exposure itself. The few provocation studies that reported a statistically significant finding often indicate a protective effect of exposure as a detrimental effect on symptoms, which might be due to chance or methodological issues [Schmiedchen et al., 2019]. Therefore, to this end, the studies that potentially reflect a true connection between EMF exposure and symptoms are insufficient as evidence that EMF hypersensitivity may occur as an environmentally inducible syndrome.

Earlier, intervention studies have been performed to test the effect of reducing EMF exposure in environments where volunteers normally experienced their symptoms [Oftedal et al., 1995, 1999; Leitgeb et al., 2008]. Although one of these [Oftedal et al., 1995] observed a possible association, this could not be replicated [Oftedal et al., 1999], and the study by Leitgeb et al. [2008] could not find any association between exposure level and sleep quality. In a recent exploratory study using an ecological momentary assessment design, Bogers et al. [2018] found weak but statistically significant correlations between perceived and actual exposure to $\mathrm{Wi}-\mathrm{Fi}$ and mobile phone antennas, namely the rate of change and number of peaks above threshold, and scores of non-specific physical symptoms (NSPS) in some but not all participants with IEI-EMF. Although two showed a positive association between EMF exposure and symptoms, two other participants showed a negative association. Using the same design, Bolte et al. [2019] reported a statistically positive association between $\mathrm{Wi}$ Fi exposure metrics (self-reported as the most significant exposure burden) and the NSPS score and symptom severity in one out of 57 participants after correcting for multiple comparisons. Even though these observations might be due to residual confounding, they may also reveal a causal link between EMF exposure and IEI-EMF symptoms in a few subjects. These scattered results, together with those of a few experimental studies, warrant further testing of the hypothesis that IEI-EMF is linked to EMF exposure.

Even though the observational and intervention studies have the advantage of considering a longer 
exposure in real life, it is impossible to have the same level of control of potential confounding factors as in provocation studies. The latter remains the most rigorous method to assess the possible electromagnetic origin of the symptoms experienced by people with IEI-EMF, as they are best suited to isolate the effects of EMF exposure. However, provocation studies should be pursued with more appropriate methodologies, as several authors have attempted in recent years using innovative methods, such as by considering the comments and suggestions of people with IEI-EMF [Huss et al., 2016; Andrianome et al., 2017; van Moorselaar et al., 2017] and by adapting the exposure system and the test environment to their conditions [van Moorselaar et al., 2017; Verrender et al., 2018]. Schmiedchen et al. [2019] emphasized that such studies should only be conducted if their methodology is carefully considered.

To improve the possibility of finding evidence of a physical effect of EMF exposure given that such an effect exists, novel approaches are warranted. An important aspect is to consider the expertise of people with IEI-EMF to a larger extent than has been done in previously published studies. This will ensure that the protocol does not only depend on researchers' preconceptions or technical constraints but also uses the experience of those that are afflicted with IEIEMF. Such an approach seems possible, as people with IEI-EMF recognize the need for well-conducted provocation studies [Prignot, 2020]. Indeed, despite the reluctance of some of them to deliberately expose themselves or to be asked to discriminate between false and true signal in double-blind conditions, many people with IEI-EMF recognize the decisive nature of the experimental evidence provided by provocation studies: they are convinced that such studies should succeed in demonstrating the "electrosomatic" nature of their disorders if the studies are appropriately conducted [Prignot, 2020]. They express a strong desire to participate.

Given this context, the ExpoComm project was implemented, with the support of the French National Research Program for Environmental and Occupational Health of Anses (EST/2017/2 RF/19). The innovative process adopted in this project involves a close collaboration between researchers and people with IEI-EMF in the development and implementation of an exposure protocol for the study of a physical link between EMF exposure and IEI-EMF symptoms. The intention was to develop a protocol with high scientific validity and acceptability for people with IEI-EMF. The protocol is applied in a provocation study with the primary aims to test whether the EMF exposure is perceived and whether real exposures result in more symptoms than sham exposures, and to test the acceptability of the protocol among the IEIEMF volunteers. This paper describes the protocol resulting from the collaborative process. The implementation of the co-development process and its results will be analyzed in another publication.

\section{METHOD}

\section{Preliminary Work}

The first step in this research was to identify the characteristics required for the protocol to be scientifically valid and innovative, and those that could be determined with participants with IEI-EMF. To this end, we assessed the latest systematic reviews of provocation studies, as well as more recent studies, in light of the experience gained by some of the authors of such studies. This preliminary work resulted in three main decisions (i) performing tests in a laboratory rather than at home to ensure adequate control of dosimetry; (ii) maintaining double-blind conditions; and (iii) characterizing the volunteers' level of sensitivity by considering their symptoms, attributions, and behaviors. The study characteristics requiring further clarification with people with IEIEMF were as follows: (i) the nature of EMF exposures: number and types of sources, exposure intensity, etc.; (ii) a study protocol allowing data analyses at the collective or individual level, the latter avoiding the problem of the possible heterogeneity of the group, but requiring more tests per volunteer to reach a sufficient statistical power; (iii) the level of standardization or individualization of the protocol; (iv) the need to reduce the anxiety-inducing nature of experimental situations; and (v) the symptoms and physiological reactions to be observed, and over what period of time.

\section{Co-Development Process}

The co-development process took the form of successive workshops involving people with IEIEMF, researchers, and professional facilitators of the Wallonia e-health Living Lab (WeLL, Liège, Belgium). The facilitators were experts in the integration of end-users in developing innovation in the health sector, having no prior knowledge of IEI-EMF. Their intervention was decided to ensure that researchers would not influence participants' decisions. Preparatory meetings were held with the researchers to clarify the basic scientific requirements.

The aims of the workshops were threefold: to verify the extent to which participants with IEI-EMF accepted the preliminary decisions, to allow them to 
express their opinions on other study characteristics, and to identify convergences and divergences in their thoughts, needs, and expectations.

\section{Participants}

Participants with IEI-EMF were recruited through contacts initiated during previous meetings and conferences, participation in previous studies, belonging to action and self-help groups, and through a building biologist. The first contact was made by email or telephone. For those interested, an in-person meeting was organized to offer more details and to answer their questions. These were very diverse, covering technical aspects on the future protocol, researchers' qualifications, potential conflicts of interest, sources of funding, the working hypothesis, etc.

Fourteen people with IEI-EMF, seven men and seven women, agreed to participate in the workshops (Table 1). Twelve participants completed the three meetings. Two participants did not attend the second workshop: one stated being too ill to attend, and the second seemed interested in IEI-EMF less as a patient than as a practitioner who wanted to share his experience as a building biologist, regularly meeting people with IEI-EMF. Positioned in such a role of expert, his involvement in the workshops proved to be hardly compatible with the co-development process initiated for people with IEI-EMF.

To carry out the discussions, a focus group method was chosen. During the first workshop, the participants were split into two groups of seven people, which appears to be the optimal size to encourage the participation of everyone and the sharing of diverse opinions [Freeman, 2006; Krueger and Casey, 2014]. This work was followed by plenary sessions to share group discussions.

The median age of our participants was 53 (37-72). Eight of them were convinced of their EHS for at least 10 years, while two participants still questioned the relationship between the experience of symptoms and EMF exposure. The subjective characteristics of their sensitivity (e.g. awareness of EHS, duration of symptoms, incriminated sources, membership of an association) varied greatly, ensuring that the group mirrored the diversity of people with IEI-EMF (Table 1).

\section{Workshops Step-by-Step}

The first workshop (W1) aimed to highlight the elements of the experimental protocol. It was structured around three main questions: What are the good reasons to participate and not to participate in a provocation study, and what would the perfect study look like? The reflection focused on the type of exposure, the place for conducting experiments, their overall organization, the parameters to observe, and other relevant aspects. Eventually, the constraints needing to be considered to ensure the scientific validity of the protocol were introduced in the discussion.

At the end of W1, it appeared that the focus group organization had not allowed the process to go as far as expected, especially regarding the constraints. A second workshop (W2) was thus organized. The participants again worked in two groups, following a more directive methodology: specific questions were raised concerning each step of the

TABLE 1. Characteristics of the Electrohypersensitivity (EHS) Participants

\begin{tabular}{|c|c|c|c|c|c|}
\hline & Age & Sex & Awareness of EHS (in years) & $\begin{array}{c}\text { Sources of exposure associated with } \\
\text { symptoms }\end{array}$ & Member of an EHS association \\
\hline EHS1 & 63 & $\mathrm{~F}$ & 10 & Wi-Fi & No \\
\hline EHS2 & 48 & M & 1 & Radar/mobile phone & No \\
\hline EHS3 & 64 & $\mathrm{~F}$ & $>10$ & $\mathrm{RF} / 50 \mathrm{~Hz}$ & No \\
\hline EHS4 & 45 & M & 4 & $\mathrm{RF} / 50 \mathrm{~Hz}$ & No \\
\hline EHS5 & 44 & M & 10 & $\mathrm{RF}$ & $\begin{array}{l}\text { Not anymore, but remains } \\
\text { informed }\end{array}$ \\
\hline EHS6 & 54 & $\mathrm{~F}$ & 3 & RF & Yes \\
\hline EHS7 & 58 & $\mathrm{~F}$ & 2 & $\mathrm{RF} / 50 \mathrm{~Hz}$ & Yes \\
\hline EHS8 & 72 & $\mathrm{~F}$ & 22 & RF & Yes \\
\hline EHS9 & 38 & M & 10 & $\mathrm{RF} / 50 \mathrm{~Hz}$ & Yes \\
\hline EHS10 & 41 & $\mathrm{~F}$ & $?$ & RF & No \\
\hline EHS11 & 47 & M & $>20$ & $\mathrm{RF} / 50 \mathrm{~Hz}$ & No \\
\hline EHS12 & 64 & $\mathrm{~F}$ & $?$ & Mobile phone/Wi-Fi & No \\
\hline EHS13 & 55 & M & 14 & $\mathrm{RF} / 50 \mathrm{~Hz}$ & Yes \\
\hline EHS14 & $(*)$ & M & $>20$ & $\mathrm{RF}$ & No \\
\hline
\end{tabular}

(*) Missing data. As a building biologist, this participant also acted as an EHS expert. 
study protocol, i.e. the journey to the test room, the repetition of real or sham exposure sessions including a rest period in the test room, a real or sham exposure period and measurements of parameters before, during, and after the exposure period, and the communication of results to the participants. After W2, a questionnaire summarizing the main points of discussion was sent to the participants, so as to confirm their choices. Workshops 1 and 2 were recorded and fully transcribed to be further analyzed.

None of the technical teams in charge of the development of the EMF exposure system participated in $\mathrm{W} 1$ and $\mathrm{W} 2$ to allow the co-development process of the study protocol to follow a course as free as possible, without emphasizing technical constraints. The conclusions of these workshops were submitted to these teams to examine the technical feasibility of a test while maintaining the acceptability criteria expressed by the participants. The protocol was then presented to them in a third workshop (W3), in the presence of a technical collaborator. The test room was shown to the participants. The detailed protocol and Hypersensitivity questionnaire were also reviewed.

\section{RESULTS}

\section{Information Gathered During the Workshops}

Accepted decisions. Decisions taken during the preliminary work were favorably received by the participants, who understood the benefit of performing the tests in the lab and the need for a double-blind design. They also recognized the need to appropriately characterize the level of sensitivity of participants.

Experimental parameters. On the basis of the analysis of the workshops and the answers to the questionnaire, parameters identified as requiring clarification with our participants, e.g. the nature of the EMF exposure, study at the collective or individual level, need to reduce the anxiety-inducing nature of exposure situations, etc., were specified. Each parameter chosen, as reported below, was approved by a majority of the participants.

Nature of exposures. The participants agreed with the use of a "cocktail" of EM signals, i.e. from multiple sources regularly encountered in occupational and residential environments, including exposure from mobile phone and DECT base stations, Wi-Fi routers, and a $50-\mathrm{Hz}$ current source.

Level of analysis. Conducting tests on an individual level is recommended [Schmiedchen et al., 2019] but requires a higher number of repetitions of real or sham exposure sessions and a higher time investment from the participants. Most participants expressed their willingness to take part in a sufficient number of tests to obtain a sufficiently high statistical power at the individual level provided that they were able to perceive the EMF exposure. Other participants declared that the required number of tests was too high. They agreed to take part in a few tests that would only reach statistical significance when aggregated at the group level. Therefore, it was decided that both options should be provided. On the basis of statistical analyses, it was determined that 12 sessions would be required to allow results at the individual level (see Statistical Analyses section) and three sessions for aggregated results (see Statistical Analyses section).

Level of protocol standardization. The individualization of the protocol regarding the durations of the rest period and exposure sessions, and the number of experimental sessions per day, was highly requested during both workshops. Furthermore, the necessity of ensuring that participants were in the right condition before starting the tests, meaning able to detect when the exposure system was really "on," was also emphasized, resulting in the development of the go/no go question (see Questionnaires section).

Anxiety reduction. Reducing anxiety associated with the test situation was considered highly appropriate. As stated by a participant, whose opinion was confirmed by others: "In my case, in fact the direct feeling, I can very easily confuse it with stress; I have great difficulty distinguishing between stress and the feeling due to exposure." To this end, the following strategies were chosen: (i) as many contacts and meetings as necessary according to the needs of each participant, before the habituation session; (ii) a habituation session where they could experiment with individualized parameters that best suited their needs, and getting to know the observer and tools used to assess the selected parameters before, during, and after the exposure period; and (iii) a pause button that allows the volunteer to stop the exposure at will, such as if the level of discomfort is too high. The individualization of the protocol showing a willingness to meet the needs of each individual could also contribute to reduce anxiety.

Symptoms, perception, and reactions to observe. In addition to subjective indicators of exposure, i.e. reported perceptions and symptoms, participants were interested in objective indicators, without giving precise indications as to what these should be. On the basis of their feedback on the effects of EMF exposure, in particular heart palpitations, more agitated or apathetic behavior, and reduced ability to concentrate, it was decided to perform three exploratory measurements: heart rate variability (through an 
electrocardiography [ECG] recording), behavioral observation during exposure time, and attention tests before, during, and after exposure time.

\section{Study Protocol}

The stepwise sequence is illustrated in Figure 1.

Recruitment and preliminary contact. Conducted by email or phone call, the aim of this first step is to present the study and confirm the eligibility of the volunteer. Based on the definition of IEI-EMF, the inclusion criteria are as follows: Being older than 18 years old; Reporting one or more non-specific symptoms (such as headaches, sleep difficulties, memory problems, mood changes, and tinnitus) attributed to any sources of EMF included in the exposure cocktail, or wondering if hypersensitive to EMF; Being able to manage one's symptoms on one's own, without the help of a relative or a health professional; Not reporting any severe diseases (cancer, COPD, Alzheimer's disease, Parkinson's disease, etc.); Not using recreational drugs considered as illegal in Belgium, where the tests take place, which could interfere with ECG and cognitive performance measurements.

First meeting. This meeting takes place at a location chosen by the volunteers. It aims to provide information on the study and discuss individualization possibilities. The informed consent document and the Hypersensitivity questionnaire (see Questionnaires section) are given at the end of the meeting. Volunteers are asked to read and complete these documents before the habituation session.

These first steps of the protocol as described in the Recruitment and Preliminary Contact and First Meeting sections are only relevant for people with IEI-EMF who were not involved in the codevelopment workshops.
Habituation session in open field (S0). S0 begins with a tour of the exposure facilities and an explanation of how the exposure system works. Afterwards, the signed consent form and the completed Hypersensitivity questionnaire are taken back. To minimize any distraction that the ECG monitoring might have on the volunteer during the real and sham exposures under double-blind conditions, a test is carried out similarly to how it is done in the later double-blind tests. Then, the volunteers are invited to rest for as long as they want in the test room. When they feel ready, the openfield exposure begins. Volunteers are allowed to switch off and on the exposure at any time by using the pause button. The duration of the exposure period is not fixed; it ends when the volunteers believe they perceive the exposure, or after $60 \mathrm{~min}$. Those who fail to perceive the exposure within 60 min or report any symptoms, even on the following evening and morning, are excluded from the study.

After this procedure, the individualization of the exposure sessions is determined by using the answers to the Hypersensitivity questionnaire and the openfield test. The base protocol (see Fig. 2) includes a single exposure session (real or sham, double-blind) per day, of an approximate duration of $40 \mathrm{~min}$ : $30 \mathrm{~min}$ of sham or real exposure followed by approximately $10 \mathrm{~min}$ of attention tests before turning off the exposure systems (tests during exposure). This protocol can be modulated depending on (i) the latency of symptoms and duration of recovery: three sessions rather than one session per day; and (ii) the severity of symptoms: adapted protocol with short exposure (approx. $5 \mathrm{~min}$, depending on volunteers' feelings) rather than the complete protocol with long exposure (approx. $40 \mathrm{~min}$ ). The four defined protocols are thus: Complete protocol 1: one session per day, each approx. $40 \mathrm{~min}$; Complete protocol 2: three sessions per day, each approx. $40 \mathrm{~min}$; Adapted protocol 1: one

\begin{tabular}{|c|c|c|c|c|}
\hline $\begin{array}{c}\text { Preliminary } \\
\text { contacts }\end{array}$ & f) $\begin{array}{c}\text { First } \\
\text { Meeting(s) }\end{array}$ & $\begin{array}{c}\text { Habituation } \\
\text { session }\end{array}$ & $\begin{array}{l}\text { Exposure } \\
\text { sessions }\end{array}$ & $\begin{array}{c}\text { Communication } \\
\text { of results }\end{array}$ \\
\hline $\begin{array}{l}\text { - Introduction } \\
\text { - Initial questions } \\
\text { - Sending of the } \\
\text { project file }\end{array}$ & $\begin{array}{l}\text { - Objectives and } \\
\text { organization } \\
\text { of the project } \\
\text { - Possible } \\
\text { individualizations } \\
\text { - Delivery of the } \\
\text { information and } \\
\text { consent document } \\
\text { and the } \\
\text { Hypersensitivity } \\
\text { questionnaire }\end{array}$ & $\begin{array}{l}\text { - Signature of the } \\
\text { consent document } \\
\text { - Presentation of } \\
\text { the test room and } \\
\text { the exposure } \\
\text { system } \\
\text { - Exposure (open- } \\
\text { field) } \\
\text { - Confirmation of } \\
\text { the protocol }\end{array}$ & $\begin{array}{l}\text { - Journey to the } \\
\text { test room } \\
\text { - Rest } \\
\text { - Go-No go } \\
\text { question } \\
\text { - Pre-exposure } \\
\text { measurements } \\
\text { - Exposure } \\
\text { (double blind) } \\
\text { - Measurements } \\
\text { during exposure } \\
\text { - Post-exposure } \\
\text { measurements } \\
\text { - Delayed } \\
\text { measurements }\end{array}$ & $\begin{array}{l}\text { - } 12 \text { sessions: } \\
\text { analysis at the } \\
\text { individual level } \\
\text { - } 3 \text { sessions: } \\
\text { aggregation of } \\
\text { results and analysis } \\
\text { at the collective level }\end{array}$ \\
\hline
\end{tabular}

Fig. 1. Study protocol. 


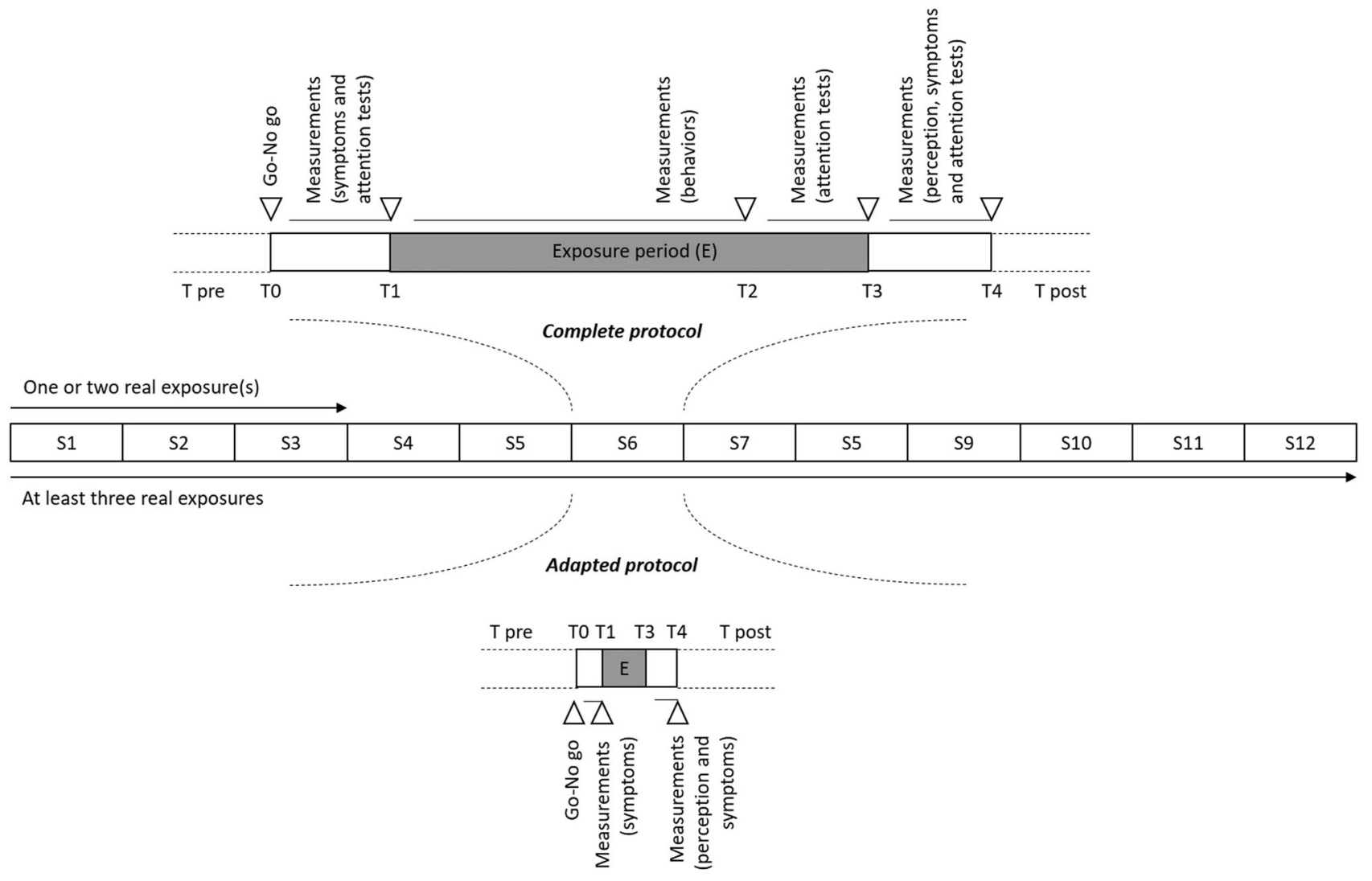

Fig. 2. Implementation of the 12 sessions of the complete and adapted protocols. S, session; E, exposure period (Complete protocol: $30 \mathrm{~min}$ of sham or real exposure followed by approx. $10 \mathrm{~min}$ of measurements; Adapted protocol: approx. $5 \mathrm{~min}$ of sham or real exposure); 12 sessions: Results analysis at the individual level; three sessions: Results analysis at the collective level.

session per day, each approx. $5 \mathrm{~min}$; Adapted protocol 2: three sessions per day, each approx. $5 \mathrm{~min}$.

If the volunteer follows an adapted protocol, only exposure perception and subjective symptoms are assessed, not other exploratory parameters (see Fig. 2). To allow volunteers enrolled in one session a day to recover appropriately between sessions, a rest period of at least 2 days (depending on how long the symptoms lasted) was selected.

Series of double-blind exposure sessions (S1 to S12). A session includes travel by car or public transport to the test room (usually less than $60 \mathrm{~min}$ ), a rest period (Tpre-T0; see Fig. 2), the go/no go question (see section Questionnaires; in T0; see Fig. 2), measurements before the exposure period (symptoms and attention tests; T0-T1; see Fig. 2), the exposure period itself (T1-T2; see Fig. 2), and measurements just before the end of the exposure period (attention tests; T2-T3; see Fig. 2) and directly after (perception, symptoms, and attention tests; T3-T4; see Fig. 2). ECG is recorded continuously during the whole exposure period for those with a complete protocol. Volunteers are also encouraged to take a rest period prior to their departure (T4-Tpost; see Fig. 2).

On arrival, before each session, the observer makes sure that the test conditions are met. Volunteers must be in a standard state with regard to their medication and other intake such as caffeine or alcohol consumption that could affect cardiac activity, cognitive performance, or symptoms. They must also answer positively to the go/no-go question. If appropriate, ECG electrodes are positioned on the volunteer and attention tests are administered. The volunteer then moves into armchair A1 (see Fig. 3a). After another facultative rest period, the observer switches the exposure system on. Then, he takes note of the volunteers' behavior during the exposure period, staying in the same position ( $\mathrm{S} 1$; see Fig. 3a) and only interacting with them to answer their questions. The standardized role of the observer is explained during the habituation session. 
(a)

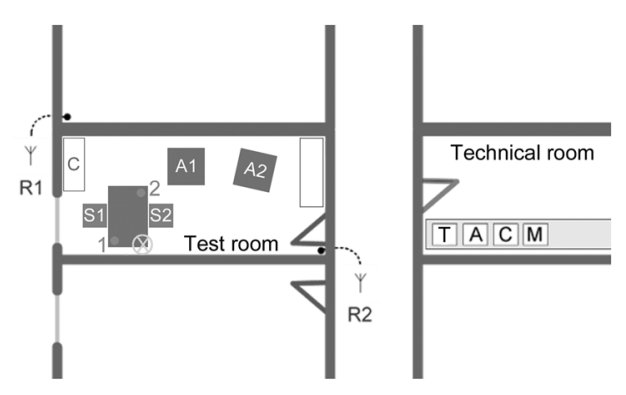

(b)

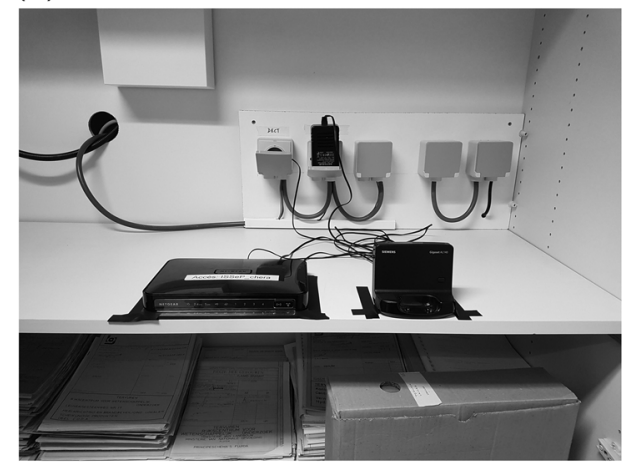

Fig. 3. (a) Exposure system: Test and technical rooms. Test room: R1, receiving antenna on the roof of the building near the mobile service antenna; R2, receiving antenna for continuous monitoring of the RF fields in the test room; $\mathrm{C}$, cupboard including the transmitting antenna, WiFi router, and DECT base station; X, halogen floor lamp; 1, ON/OFF control button positioned on the desk; 2, pause button of the system positioned on the desk; $\mathrm{A} 1$, test armchair at $2 \mathrm{~m}$ from the RF sources; A2, armchair at $3 \mathrm{~m}$ from the RF sources; $\mathrm{S} 1$, seat of the observer; $\mathrm{S} 2$, seat of the volunteer during the attention tests. Technical room: T, 50-Hz transformer; A, amplifier and attenuator; $\mathrm{C}$, computer; $\mathrm{M}$, radiofrequency measuring instrument connected to the receiving antenna R2. (b) Exposure system: RF exposure devices in C.

The protocol includes a double-blind procedure and randomization of sham and real exposures: volunteers are assigned to a first trio of sham or real exposures (randomization based on a computergenerated series) to avoid bias related to the exposure sequence [Eltiti et al., 2007; Schmiedchen et al., 2019]. This results in the inclusion of volunteers in one of the six randomized permutations. The randomization is ensured for the remaining nine sessions. The volunteers are told that during the first three sessions, they will be really exposed at least once, possibly twice. For the 12 sessions, volunteers are told that they will be really exposed at least three times.

Communication of results. The workshops showed that participants were somewhat undecided as to their preference for individual or collective communication of the results. It was finally decided that individual results including exposure data, perceptions, and symptoms, as well as exploratory measurements, would be shared with volunteers during individual interviews while the collective results would be communicated during a focus group after all tests had been conducted.

\section{Exposure System}

The exposure system is split into two rooms as described in Figure 3a. They are called hereafter "Test room" and "Technical room."
Description of the exposure system and premises. Four types of EMF can be produced, either separately or simultaneously. In the protocol defined with the workshop participants, an EMF cocktail was chosen, so the sources are switched on simultaneously. The signals from the second, third, and fourth generation mobile service antennas (located on the roof of the building in which the technical and test rooms are located) are picked up by a nearby receiving antenna (R1) which is also located on the roof. These signals are transmitted, by means of a coaxial cable, to a programmable attenuator (RCDAT-6000-30 USB/ ETH; Mini-Circuits, Brooklyn, NY) and an amplifier (A) (HPA-25W-272+ HPA Rack Mount Amplifier RoHS, Mini-Circuits) located in the technical room. Another coaxial cable connects the amplifier output to a directional transmitting antenna dissimulated in a cupboard $(\mathrm{C} 1)$ in the test room. The transmitting antenna is a SENCITY Cube from Hubert \& Suhner (Pfäffikon, Switzerland), which is designed for inbuilding coverage and operates in the frequency range from 698 to 2,700 MHz. The beam of this antenna is oriented towards the armchairs (A1) and (A2) to maximize the field levels in that direction. A Wi-Fi router and DECT cordless phone base station are also dissimulated in the cupboard ( $\mathrm{C}$ in Fig. $3 \mathrm{a}$ and $\mathrm{b}$ ) and are controlled and powered from the technical room. A magnetic field at $50 \mathrm{~Hz}$ is produced by a current loop consisting of several copper wire turns placed above the false ceiling of the test room, so that the 
volunteer's head is exposed to a higher magnetic flux density than the lower body parts. This loop is powered by a small transformer (T) located in the technical room. The $50-\mathrm{Hz}$ rms electrical current delivered in the loop is fixed, as is the rms magnetic flux density in the test room. All the above-mentioned EMF sources are controlled by a computer and specific software developed for this application.

This exposure system has been designed to generate EMF levels comparable to those found in places such as offices equipped with Wi-Fi routers and/ or DECT cordless phone base stations or in any house close to mobile phone base stations. The rooms are located in a sparsely used professional building. In the surrounding rooms, there are no "GSM or DECT users" and the unwanted Wi-Fi signals are weak and most of the time in the standby state. There are neither transformers nor power cables in the neighboring rooms. To avoid sources of unintentional fields related to LED or compact fluorescent/neon lighting, natural light and a halogen floor lamp are used and provide sufficient brightness for testing and questionnaire administration. Further means have been implemented to mitigate radiation in the test room from other sources located inside or outside the building: a protective "anti-wave film" has been applied on the window of the test room and also on the window of the neighboring room and a conductive foil has been placed under the floor. The objective was to reach average levels less than $0.1 \mathrm{~V} / \mathrm{m}$ for radiofrequency EMF and less than $0.05 \mu \mathrm{T}$ for $50-\mathrm{Hz}$ magnetic field. The test room is not strictly speaking a radiation-free zone, but as shown in Table 2, the levels of exposure are low and constant. This is a compromise reached with our workshop participants to address other requirements, especially the need for a window providing natural light and fresh air between two test sessions. The temperature and humidity levels in the test room are recorded immediately before and after each session.
When the volunteer feels ready, the observer activates the exposure system with a general switch ("ON/OFF" control button). When the exposure is real, above the armchair A1, at the approximate position of the volunteer's head, i.e. $1 \mathrm{~m}$ above the ground (see Fig. 4), the radiofrequency field strength is between 1.4 and $1.5 \mathrm{~V} / \mathrm{m}$ and the magnetic flux density is $0.75 \mu \mathrm{T}$ (see Table 2). The real or sham nature of the exposure is determined automatically by the computer. Afterwards, the volunteer can deactivate and reactivate the exposure system at will with the pause button (2 in Fig. 3a), such as if his level of discomfort is too high.

The test room is furnished to make it welcoming and relaxing. All furniture, including two comfortable armchairs, a table, and two chairs, is made of wood. As requested by a few workshop participants during the workshops, a grounded steel plate is placed in the test room and a water station is accessible nearby, allowing them to "cool down" and "discharge" if necessary.

Quality control of the exposure system. Doubleblind exposure: pre-tests have established that the exposure system does not give audible or visible cues of its status. Its design ensures that the nature of each exposure session remains unknown to the participants and the observer until the completion of the individual or collective protocol.

Exposure monitoring: the radiofrequency EMF exposure comes from picked-up signals from mobile service antennas, a DECT station, and Wi-Fi router. As a result, the EMF exposure varies in time and should be monitored. Every $15 \mathrm{~s}$, the radiofrequency EMF strength in the test room is continuously recorded by a field meter (M) located in the technical room, connected to a receiver antenna (R2) placed in the test room. The functioning of the exposure system is tested before each session.

TABLE 2. Levels of EMF Exposure Measured in the Test Room in A1 (1 m Above the Floor)

\begin{tabular}{|c|c|c|c|c|}
\hline & \multicolumn{2}{|c|}{ Exposure system OFF } & \multicolumn{2}{|c|}{ Exposure system ON } \\
\hline & rms max average & rms average & rms max average & rms average \\
\hline Mobile phone antennas $(\mathrm{V} / \mathrm{m})$ - All signals & $0.08-0.1$ & 0.06 & 1.5 & $1.4-1.5$ \\
\hline LTE 800 & 0.06 & 0.04 & 1.5 & $0.7-1$ \\
\hline 2G-UMTS 900 & 0.05 & 0.04 & 0.8 & $0.6-0.7$ \\
\hline LTE 1800 & 0.03 & 0.02 & 0.7 & $0.4-0.5$ \\
\hline LTE 2100 & $<0.02$ & 0.01 & 0.3 & $0.1-0.2$ \\
\hline Wi-Fi box (V/m) $(2.4 \mathrm{GHz})$ & 0.06 & 0.01 & 0.2 & 0.1 \\
\hline Wi-Fi box $(\mathrm{V} / \mathrm{m})(5 \mathrm{GHz})$ & 0 & 0 & 0.1 & 0.03 \\
\hline DECT base station $(\mathrm{V} / \mathrm{m})(1897 \mathrm{MHz})$ & 0.06 & $<0.02$ & 1.6 & 0.2 \\
\hline $50 \mathrm{~Hz}(\mu \mathrm{T})$ & $<0.05$ & $<0.05$ & 0.75 & 0.75 \\
\hline
\end{tabular}




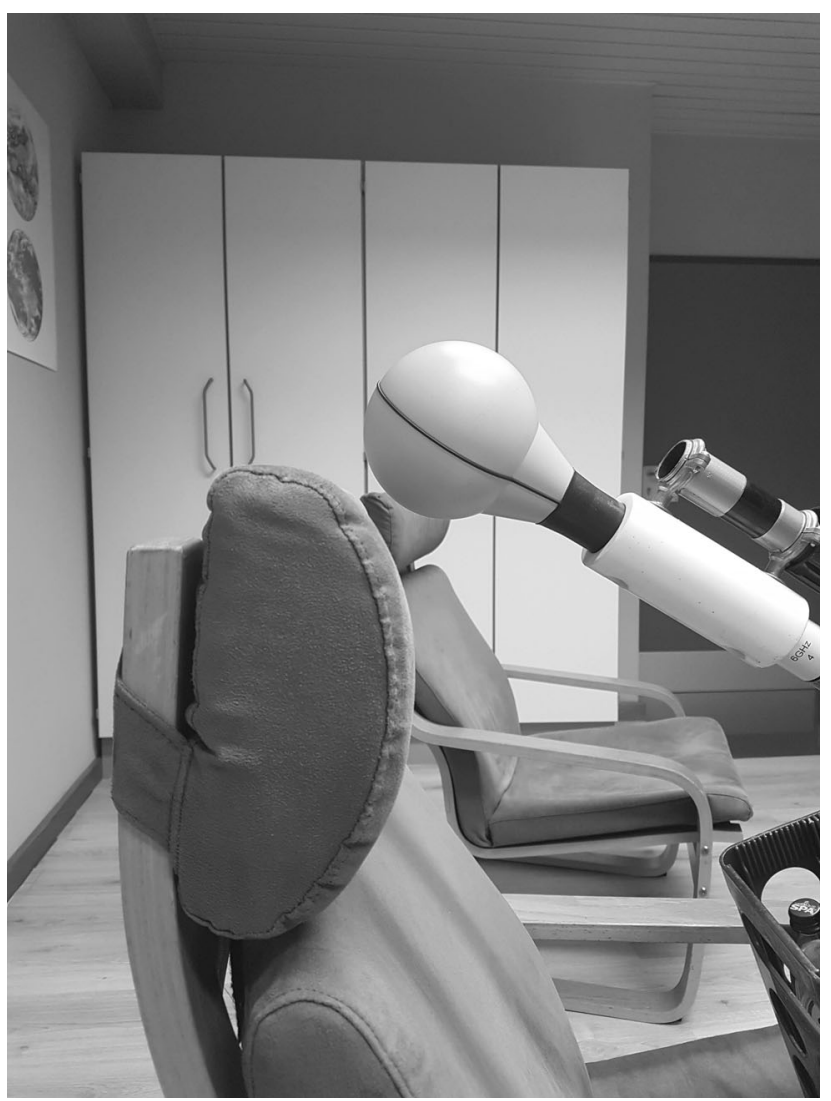

Fig. 4. Positioning of the radiofrequency meter probe when measuring in $\mathrm{A} 1$.

EMF spatial variations: as the volunteer can move during the test, the 6-min average rms values of the radiofrequency field strength, as well as the rms magnetic flux density, have been measured at different locations inside the test room. The spatial variation of the radiofrequency field strength is between 1.0 and $1.5 \mathrm{~V} / \mathrm{m}$ while the magnetic flux density remains equal to $0.75 \mu \mathrm{T}$ under the wire loop.

\section{Tools Used and Developed}

Questionnaires. Hypersensitivity questionnaire: it is composed of seven sections devoted to EMF risk perception, sources of exposure-triggering symptoms and general level of EMF sensitivity, behavioral and social consequences, symptoms in case of exposure (EHSI) [Nordin et al., 2013], current health state (PHQ-15, PHQ-9, GAD-7) [Kroenke et al., 2001, 2002; Spitzer et al., 2006], and general sensitivity (Tellegen absorption scale and Highly Sensitive Person scale) [Tellegen and Atkinson, 1974; Aron and Aron, 1997]. For comparison purposes, this questionnaire is designed to be completed by any person, whether reporting to be suffering from IEI-EMF or not. As more than 3 months could elapse between the habituation session and the last exposure session, and given the possible evolution of IEI-EMF over time [Röösli et al., 2010], the hypersensitivity questionnaire is administered three times: after inclusion, after the completion of 3 or 12 tests, depending on the protocol, and after the communication of collective results. This allows for distinguishing between the effects of study participation and of the communication of results on the level of IEI-EMF.

Factors affecting cardiac activity and cognitive performance: Volunteers are asked to report any previous pathologies such as head injury, cerebrovascular accident, neurological disease, heart surgery, etc., as well as medication, tobacco, alcohol, and caffeine consumption. This questionnaire is administered at the beginning of the habituation session.

Description of the neutral state or normal reactivity state: During the workshops, the participants described periods when they were less sensitive to EMF, especially when they felt particularly "charged." To avoid such situations, participants are asked during S0 to describe their state of normal reactivity. Their answers are used to construct the go/ no go question asked before starting an exposure session.

Acceptability questionnaire: It includes three questions concerning the appropriateness of the study to investigate IEI-EMF, on a scale from 0 to 10 , the relevance of various characteristics of the protocol, and their impact on the participants' willingness to take part in the study, on a 4-point scale. This questionnaire is administered after S0, $\mathrm{S} 1, \mathrm{~S} 3, \mathrm{~S} 12$, and the communication of collective results.

Measurements. Subjective and objective parameters are recorded. The former includes exposure perception and symptoms reporting (EHSI questionnaire) [Nordin et al., 2013], to be assessed immediately before and after the exposure sessions. The volunteers record the perception of exposure by choosing "I was really exposed" or "I was not really exposed." In addition, they specify the level of certainty of their choice on a scale of 0 to 10 . The volunteers are invited to fill in the EHSI questionnaire in the evening and the next morning following one exposure session per day. Other participants, whose reactions are supposedly quicker and who follow three exposure sessions per day, do not complete this questionnaire.

Additionally, for subjects exposed for at least $30 \mathrm{~min}$, the following objective parameters are explored: (i) levels of concentrated attention 
(d2 test) [Brickenkamp and Zillmer, 1998] and selective attention (Stroop test) [Stroop, 1935] before, during, and after the exposure period; (ii) heart rate variability; and (iii) behaviors during the test. Volunteers are allowed to stand up and move in the room. The observer records their position and movements, as well as their comments.

The ECG recording system is a class IIa medical device [Medical Devices Directive, 1993] tested for electromagnetic compatibility. Low pass filtering cut-off frequency is set to $40 \mathrm{~Hz}$ with an added notch at $50 \mathrm{~Hz}$. To ensure double-blind analyses, pre-tests were performed and confirmed that no sign of activation of the EMF was visible on the displayed ECG signal.

\section{Statistical Analyses}

As a study aimed at evaluating the relevance of the protocol developed and its acceptability to IEIEMF volunteers, analyses are planned at the individual level (principal objective) and at the collective level (secondary objective).

Principal objective: analyses at the individual level. Concerning the perception of real exposure, the session with the lowest degree of certainty is removed from the analysis (or the two sessions, in case of equality). For the protocol allowing consideration of sessions as independent, statistical power was calculated with GPower 3.1 (HeinrichHeine-Universität, Düsseldorf, Germany) on the basis of the remaining 11 or 10 sessions. In the unlikely event that the uncertainty is equal for three or more sessions, no sessions are removed from the analysis to ensure a sufficient statistical power.

At present, there are no known values for the magnitude of the effect size associated with exposure of individuals with IEI-EMF. We tested the probability of observing a proportion of 0.91 or 0.9 (in case of removing one or two sessions, respectively) of correct responses in a chance distribution (around $0.5)$, considering a univariate approach. As our hypothesis is that people with IEI-EMF should obtain higher scores than by chance, we used a one-tailed analysis. We assumed effect sizes of 0.41 or 0.4 and applied a significance level of 0.05 , resulting in a statistical power of 0.8 . As a consequence, participants with more than one misperception in their 10 or 11 remaining sessions are considered as having failed to distinguish between real and sham exposure.

Descriptive statistics are used to summarize the number of symptoms experienced before and after the exposure periods with real and sham exposures, respectively.

\section{Secondary Objective: Analyses at the Collective Level}

For analyzing perception and symptoms, the first three sessions from each individual are applied. The increase in the number of symptoms reported before and after exposure are compared between real and sham sessions by $\chi^{2}$ tests.

For perception, a variable combining perceived exposure (yes or no) and the level of certainty of exposure are constructed and compared by unpaired $t$ test. It goes from -10 (full certainty of no exposure) to +10 (full certainty of exposure). If this analysis results in a statistically significant finding, we explore which of the two "components" is important for the finding. Perceived exposure is analyzed by $\chi^{2}$ tests. The level of certainty of being exposed is compared by unpaired $t$ tests.

As a complementary analysis, the impact of the chosen protocol (complete or adapted, see Habituation Session in Open Field section) on the combined variable is examined by including the protocol type as a covariate in the model using Analysis of Covariance.

For homogeneity purposes, the above analyses is performed only on subjects who are not questioning whether they have IEI-EMF, based on the Hypersensitivity questionnaire. The other subjects are only analyzed at the individual level. Additional $t$ tests are used to assess the relevance of the exploratory variables described in the Measurements section in assessing the sensitivity of individuals. To test for a period effect, e.g. as a result of habituation, for participants conducting 12 sessions, we analyze the relation between the period and responses (subjective and physiological ones).

Eventually, the acceptability of the protocol is tested by applying the Acceptability questionnaire and by comparing IEI-EMF volunteers who participated in the co-development group with those who did not. On one hand, a $t$ test is applied on the question examining the appropriateness of the study. On the other hand, a Wilcoxon test is applied on the relevance and willingness questions, item per item and on a global score compiling all the items. A Friedman test is applied to compare the item's scores on the same subjects at the different stages of the study.

Sample size: Considering a medium effect size of 0.5 and provided a significance level of $0.05,30$ participants with IEI-EMF, with three sessions each, are required to achieve a statistical power of 0.8 for a $t$ test (GPower 3.1). 


\section{Ethical Considerations}

The study was approved by the Ethics Committee of Erasmus-Université Libre de Bruxelles (P2019/114). Informed written consent was obtained from all volunteers.

\section{DISCUSSION}

The ExpoComm project introduced several innovations, resulting from the involvement of people with IEI-EMF, and focusing on the attenuation of the anxiogenic nature of the tests, individualization of the protocol, validation of the normal reactivity state before the tests, and the use of a cocktail of real, rather than artificially generated, EMF exposures. The objective of involving people with IEI-EMF was to increase the relevance and acceptability of the protocol, while respecting technical constraints and scientific requirements. With regard to effectiveness, it is necessary to rely on the experiential knowledge of individuals with IEI-EMF to adjust the protocol to the perceived characteristics of their sensitivity, and to maximize the probability of highlighting it.

After the first workshop, the participants stated that they were pleased to have the opportunity to share their experience and collaborate in the development of a scientific study of IEI-EMF. Despite this enthusiasm, it was not always easy for them to determine their needs. This led to a maximalist approach (a cocktail with as many exposures as possible, long periods of exposure and rest, etc.) which is not the easiest to implement. The compensation for this might be a higher level of confidence from the participants, resulting from the involvement of people with IEIEMF in the protocol development, and visible efforts to cater to their needs.

Participating in a provocation test is a source of anxiety for people with IEI-EMF: it involves deliberate exposure to a phenomenon perceived as harmful, and capable of causing painful or disabling symptoms. Test procedures can further amplify this anxiety, such as the design of the experiment, the information given to subjects, and the attitude of the researchers. Symptoms and sensations created by stress and anxiety may mask subtler sensory or physiological reactions to real exposure. It therefore seemed crucial for us to reduce this anxiety. Finally, the level of sensitivity of individuals is assessed on the basis of their definition of their neutral state or their normal EMF reactivity. This is a crucial step in the protocol as it is on the basis of this definition that the volunteer determines before each session whether he or she is in the right condition to start a session. The relevance of these strategies is under continuous evaluation through the Acceptability questionnaire. Controlled exposure tests will be conducted until summer 2020 . First results are expected in late 2020.

\section{ACKNOWLEDGMENTS}

We would like to express our warmest thanks to the people with IEI-EMF who participated in the workshops with great enthusiasm and to the people who helped us in the recruitment process.

We also thank Wallonia e-health Living Lab (WeLL), whose expertise in integrating end-users into the development of innovations in the health sector ensured the successful conduct of the study, and Dr. Anke Huss for sharing information on their study with mobile exposure units. Many thanks also to the team Biostatistiques du Pôle Santé (BIOPS-ULB), who provided us with information regarding statistical analyses.

Maryse Ledent, Christophe Geuzaine, Véronique Beauvois, Catherine Bouland, and Luc Verschaeve are members of the Belgian BioElectroMagnetics Group. This research group is partly supported by ELIA TSO, under a contract of scientific independence.

\section{REFERENCES}

Andrianome S, Gobert J, Hugueville L, Stéphan-Blanchard E, Telliez F, Selmaoui B. 2017. An assessment of the autonomic nervous system in the electrohypersensitive population: A heart rate variability and skin conductance study. J Appl Physiol 123:1055-1062.

Anses. 2018. Hypersensibilité électromagnétique ou intolérance environnementale idiopathique attribuée aux champs électromagnétiques. Rapport d'expertise collective. AVIS de l'Agence nationale de sécurité sanitaire de l'alimentation, de l'environnement et du travail [in French].

Aron EN, Aron A. 1997. Sensory-processing sensitivity and its relation to introversion and emotionality. J Pers Soc Psychol 73:345-368.

Baliatsas C, Bolte J, Yzermans J, Kelfkens G, Hooiveld M, Lebret E, van Kamp I. 2015. Actual and perceived exposure to electromagnetic fields and non-specific physical symptoms: An epidemiological study based on self-reported data and electronic medical records. Int $\mathbf{J}$ Hyg Environ Health 218:331-344.

Bogers RP, van Gils A, Clahsen SCS, Vercruijsse W, van Kamp I, Baliatsas C, Rosmalen JGM, Bolte JFB. 2018. Individual variation in temporal relationships between exposure to radiofrequency electromagnetic fields and non-specific physical symptoms: A new approach in studying 'electrosensitivity. Environ Int 121:297-307.

Bolte JFB, Baliatsas C, Eikelboom T, van Kamp I. 2015. Everyday exposure to power frequency magnetic fields and associations with non-specific physical symptoms. Environ Pollut 196:224-229. 
Bolte JFB, Clahsen S, Vercruijsse W, Houtveen JH, Schipper CMA, van Kamp I, Bogers R. 2019. Ecological momentary assessment study of exposure to radiofrequency electromagnetic fields and non-specific physical symptoms with self-declared electrosensitives. Environ Int 131:104948.

Brickenkamp R, Zillmer E. 1998. The d2 Test of Attention. Seattle, WA: Hogrefe \& Huber Publishers. p 72.

de Graaff B, Bröer C. 2012. 'We are the canary in a coal mine': Establishing a disease category and a new health risk. Health Risk Soc 14:129-147.

Dieudonné M. 2016. Does electromagnetic hypersensitivity originate from nocebo responses? Indications from a qualitative study: IEI-EMF and the Nocebo Response. Bioelectromagnetics 37:14-24.

Dieudonné M. 2019. Becoming electro-hypersensitive: A replication study. Bioelectromagnetics 40:188-200.

Eltiti S, Wallace D, Ridgewell A, Zougkou K, Russo R, Sepulveda F, Mirshekar-Syahkal D, Rasor P, Deeble R, Fox E. 2007. Does short-term exposure to mobile phone base station signals increase symptoms in individuals who report sensitivity to electromagnetic fields? A double-blind randomized provocation study. Environ Health Perspect 115:1603-1608.

Freeman T. 2006. "Best practice" in focus group research: Making sense of different views. J Adv Nurs 56:491-497.

Genuis SJ, Lipp CT. 2012. Electromagnetic hypersensitivity: Fact or fiction? Sci Total Environ 414:103-112.

Huss A, Murbach M, van Moorselaar I, Kuster N, van Strien R, Kromhout H, Vermeulen R, Slottje P. 2016. Novel exposure units for at-home personalized testing of electromagnetic sensibility: Novel exposure units for at-home personalized testing. Bioelectromagnetics 37:62-68.

Kroenke K, Spitzer RL, Williams JBW. 2001. The PHQ-9: Validity of a brief depression severity measure. J Gen Intern Med 16:606-613.

Kroenke K, Spitzer R, Williams J. 2002. The PHQ-15: Validity of a new measure for evaluating the severity of somatic symptoms. Psychosom Med 64:258-266.

Krueger RA, Casey MA. 2014. Focus Groups: A Practical Guide for Applied Research, Fifth edition. Thousand Oaks, CA: SAGE Publications. p 280.

Leitgeb N, Schröttner J, Cech R, Kerbl R. 2008. EMF-protection sleep study near mobile phone base stations. Somnologie Schlafforschung Schlafmed 12:234-243.

McCarty DE, Carrubba S, Chesson AL, Frilot C, Gonzalez-Toledo E, Marino AA. 2011. Electromagnetic hypersensitivity: Evidence for a novel neurological syndrome. Int J Neurosci 121:670-676.

Medical Devices Directive. 1993. 93/42/EEC MDD and subsequent amendments Annex IX, Rule 10: Active devices intended to allow direct diagnosis or monitoring of vital physiological processes. Available from https://eur-lex.europa.eu/legalcontent/EN/TXT/?uri=CELEX:01993L0042-20071011 [last accessed 10 June 2020].

van Moorselaar I, Slottje P, Heller P, van Strien R, Kromhout H, Murbach M, Kuster N, Vermeulen R, Huss A. 2017. Effects of personalised exposure on self-rated electromagnetic hypersensitivity and sensibility-A double-blind randomised controlled trial. Environ Int 99:255-262.
Nordin S, Palmquist E, Claeson A-S, Stenberg B. 2013. The environmental hypersensitivity symptom inventory: Metric properties and normative data from a population-based study. Arch Public Health 71:18.

Oftedal G, Nyvang A, Moen BE. 1999. Long-term effects on symptoms by reducing electric fields from visual display units. Scand J Work Environ Health 25:415-421.

Oftedal G, Straume A, Johnsson A, Stovner L. 2007. Mobile phone headache: A double blind, sham-controlled provocation study. Cephalalgia 27:447-455.

Oftedal G, Vistnes AI, Rygge K. 1995. A double blind study of skin symptoms after reduction of electric fields from VDUs. Scand J Work Environ Health 21:335-344.

Prignot N. 2020. Le procès d'une maladie: l'électrosensibilité au banc des accusés. In: Lefève Céline, editor, François Thoreau et Alexis Zimmer (éditeurs), Les humanités médicales. L'engagement des sciences humaines et sociales en médecine, (collection La personne en médecine). Paris, France: Doin. pp 203-212.

Regel SJ, Negovetic S, Röösli M, Berdiñas V, Schuderer J, Huss A, Lott U, Kuster N, Achermann P. 2006. UMTS base station-like exposure, well-being, and cognitive performance. Environ Health Perspect 114:1270-1275.

Röösli M, Mohler E, Frei P. 2010. Sense and sensibility in the context of radiofrequency electromagnetic field exposure. Comptes Rendus Phys 11:576-584.

Rubin GJ, Hillert L, Nieto-Hernandez R, van Rongen E, Oftedal G. 2011. Do people with idiopathic environmental intolerance attributed to electromagnetic fields display physiological effects when exposed to electromagnetic fields? A systematic review of provocation studies. Bioelectromagnetics 32:593-609.

Rubin GJ, Nieto-Hernandez R, Wessely S. 2010. Idiopathic environmental intolerance attributed to electromagnetic fields (formerly 'electromagnetic hypersensitivity'): An updated systematic review of provocation studies. Bioelectromagnetics 31:1-11.

Schmiedchen K, Driessen S, Oftedal G. 2019. Methodological limitations in experimental studies on symptom development in individuals with idiopathic environmental intolerance attributed to electromagnetic fields (IEI-EMF)—A systematic review. Environ Health 18:88.

Spitzer RL, Kroenke K, Williams JBW, Löwe B. 2006. A brief measure for assessing generalized anxiety disorder: The GAD-7. Arch Intern Med 166:1092-1097.

Stroop JR. 1935. Studies of interference in serial verbal reactions. J Exp Psychol 28:643-662.

Tellegen A, Atkinson G. 1974. Openness to absorbing and self-altering experiences ("absorption"), a trait related to hypnotic susceptibility. J Abnorm Psychol 83:268-277.

Verrender A, Loughran SP, Anderson V, Hillert L, Rubin GJ, Oftedal G, Croft RJ. 2018. IEI-EMF provocation case studies: A novel approach to testing sensitive individuals: IEI-EMF provocation case studies. Bioelectromagnetics 39:132-143.

World Health Organization. 2007. Environmental Health Criteria 238: Extremely Low Frequency Fields. Geneva, Switzerland: World Health Organization. 